\title{
Aspekte von Gender in der Sprach(aus)bildung Deutsch (als zweite, dritte und Folgesprache)
}

Kristina Peuschel

\section{Einleitung}

Geschlecht und Sprache markieren und positionieren Menschen. Beide Kategorien werden neben anderen als bipolare Differenzlinien sozial- und erziehungswissenschaftlicher Perspektiven angesehen (vgl. Leiprecht/Lutz 2015: 287). Während Sprache in ihrer Ausprägung als Zweit-, Fremd- und Folgesprache und in Abgrenzung zur Erstsprache Deutsch Hauptgegenstand der Fächer Deutsch als Fremdsprache (DaF) und Deutsch als Zweitsprache (DaZ) ist, wird der Kategorie Geschlecht bzw. Gender als analytischer Kategorie im wissenschaftlichen Diskurs unserer Fächer bisher wenig Aufmerksamkeit geschenkt. Unterschiedliche Perspektivierungen auf Deutsch (als Zweitsprache, als Fremdsprache) in den gegenwärtigen Migrationsgesellschaften (vgl. Mecheril 2004: 8) lassen das Einschreiben der Kategorie Gender und der damit verbundenen genderreflexiven Denk- und Forschungsansätze durchaus zu. Um mögliche wechselseitige Bezugnahmen zu konkretisieren, ohne voreilig theoretisch, empirisch oder praxisbezogen Position zu beziehen, nähert sich der vorliegende Beitrag dem Themenkomplex „Gender und Sprache in DaZ*, DaF und Sprachbildung“" an, zunächst über das Aufzeigen der Absenz, anschließend über einen Forschungsüberblick und abschließend über Desiderata und offene Fragen für zukünftige Betrachtungen. Ziel des Beitrages ist es, im Rahmen der Auseinandersetzung um und ggf. Neuausrichtung der normativen Grundlagen und reflexiven Verortung unserer Fächer Gender als eine der zentralen Kategorien zu setzen, die sie gesellschaftlich ist. 


\section{Perspektiven öffnen gegen die Absenz - Gender in der Fremdsprachendidaktik}

Von der Marginalität der analytischen Kategorie Gender in Bezug auf Fremdsprachenunterricht, Fremdsprachenforschung, Zweit- und Fremdspracherwerb zeugt u. a. das langjährige vollkommene Fehlen von Basisartikeln zum Themenkomplex Gender in den großen deutschsprachigen Handbüchern wie dem Handbuch Fremdsprachenunterricht (Bausch/Christ/Krumm 2007) oder dem zweibändigen Standardwerk Deutsch als Fremd- und Zweitsprache - Ein internationales Handbuch (Krumm et al. 2010). Von der wiederkehrenden oder auch fortschreitenden Bedeutung von Gender im Kontext institutioneller Fremdsprachenvermittlung hingegen zeugen die Aufnahme eines Kapitels „Geschlecht" (Schmenk 2016) in die Neuauflage des Handbuch Fremdsprachenunterricht (Burwitz-Melzer et al. 2016), ebenso wie Themenhefte fachspezifischer Praxiszeitschriften wie z. B. das Themenheft „Negotiating Gender" (Der fremdsprachliche Unterricht. Englisch 135/2015), das Themenheft „Jungen fördern" (Der fremdsprachliche Unterricht. Französisch 122/2013), das Themenheft „Gender" (Praxis Fremdsprachenunterricht 6/2009) sowie das kürzlich erschienene Studienbuch Gender und Language Learning - Research and Practice (Elsner/Lohe 2016). Für die Fächer Deutsch als Fremdsprache und Deutsch als Zweitsprache liegen bisher keine Themenhefte zu Gender in den überregional rezipierten Praxiszeitschriften wie zum Beispiel Fremdsprache Deutsch vor.

An wissenschaftlichen Publikationen stehen einige wenige Einzeldarstellungen, Sammelbände und Forschungsüberblicke in internationalen Zeitschriften zur Verfügung, so Sunderland (2000) Pavlenko et al. (2001), Decke-Cornill/Volkmann (2007a), Schmenk (2009), wovon allein Schmenk (2009) eine deutschsprachige Monographie ist. Grundsätzlich fällt auf, dass sich wenige Einzelpersonen ausgewählter Fragestellungen rund um Aspekte von Gender annehmen, Forschungsergebnisse und Praxismodelle entwickeln, diese jedoch kaum breit rezipiert werden und von anderen Themen und Fragestellungen auf eine Randposition im Fachdiskurs verwiesen werden. Während fachintern die Forschungslage und damit möglicherweise auch die Reflexionstiefe zu Gender in der Breite als unbefriedigend bezeichnet werden kann, liefern erziehungswissenschaftliche Studien interessante Ergebnisse (vgl. z. B. Faulstich/Weber/Willems 2009²). Die intensivste Auseinandersetzung um Sprache und Gender findet jedoch in den Gender Studies statt (vgl. z. B. Schößler 2008, Hornscheidt 2013), allerdings mit bisher geringen Auswirkungen auf fachinterne Fragestellungen für DaZ*, DaF und Sprachbildung. Angesichts dieser auffällig kurzen Zusammenstellung stellt sich die 
Frage, warum gerade für DaZ und DaF bisher kein Themenheft zu Gender vorliegt bzw. aus welchen Gründen Gender in diesen Fächern kaum wissenschaftlich thematisiert wird. Worin liegt diese Absenz, die auch als De-Thematisierung interpretiert werden könnte, begründet? Schmenk (2007), die sich ähnliche Fragen in Bezug auf die Fremd- und Zweitsprachenforschung gestellt hat, antwortet hierzu:

\section{(...) gender is a category that is extraordinary difficult to study in our field. Not because it is not important, not because it does not play a role; but because there are certain „truths" in and about the field that have long been established and are continuously reproduced. (Schmenk 2007: 123)}

Gender gilt als universale Kategorie menschlicher und gesellschaftlicher Interaktion, Identifikation und Wissensproduktion (vgl. Schößler 2008: 14f.). Gleichzeitig ist das Sprechen über die Kategorie und ihre Wirkungen stark von Alltagswissen geprägt, in dem die Sprecher*innen häufig die Differenzen zweier wahrgenommener Geschlechter betonen. In Bezug auf das Fremdsprachenlernen sei auch hier das Beispiel angeführt, dass Mädchen grundsätzlich gut in Sprachen seien, deshalb bevorzugt schulische Sprachfächer und weniger MINT-Fächer belegten, damit in ihrer Studien- und Berufswahl vorgeprägt seien und dann sprachintensive Berufe ergriffen. Diese alltagsbasierten „beliefs“ (vgl. Schmenk 2007: 121ff.) würden durch empirische Studien, die Geschlechterdifferenzen unhinterfragt übernehmen, konstant bestätigt. Auch ausgewählte Ergebnisse der großen bildungswissenschaftlichen Schulleistungsstudien wie PISA tragen dazu bei, geschlechterdifferenzierend festzustellen, dass Jungen beispielsweise durchschnittlich schlechter lesen als Mädchen (vgl. Prenzel et al. 2013: 232). In alltagsbezogenen und auch empirischen Beispielen wird Geschlecht als anatomische, dichotome Kategorie gesetzt, die seit den 1970er Jahren mit sex benannt wird und die mit (biologisch) männlich/weiblich zwei Gegenpole vermeintlich unveränderlich gegenüberstellt. Dies entspricht in Bezug auf Gender als analytischer Kategorie dem Reflexionsstand der 1970er Jahre. Ein Grund für dieses zurückhaltende Aufgreifen aktueller Gendertheorien liegt möglicherweise in der Ausdifferenzierung akademischer Disziplinen in den 1970er Jahren. So schreibt Fleßner (2015: 312):

Geschlechterbewusste Pädagogik und interkulturelle Pädagogik bzw. Migrationspädagogik weisen (...) Parallelen in ihrer Geschichte auf. Beginnend in den 1970er Jahren und konsolidiert in den 1980er Jahren haben beide ihre Wurzeln 
in der Auseinandersetzung mit sozialer Ungleichheit und gesellschaftlicher Differenz' (ebd.).

Auch das Fach Deutsch als Zweitsprache konsolidiert sich in der Auseinandersetzung mit sozialer Ungleichheit auf Grund sprachlicher Differenz. Während sich also in der einen Disziplin Gender gewidmet wurde, wurden in der anderen Zweitsprachenerwerb, zweitsprachliche Sprachsysteme, Interkulturalität etc. bearbeitet. Thematische Verbindungen wurden bisher wenig betrachtet bzw. stärker in anderen Disziplinen wie Kulturwissenschaften oder Gender Studies reflektiert - zum Nachteil, wie ich finde, unserer Fächer.

Im folgenden Abschnitt wird daher versucht einen weitgehend fachspezifischen Überblick über Forschungsergebnisse und theoretische Auseinandersetzungen zum Themenfeld Gender und Sprache(n) lernen zu geben und interdisziplinäre Verbindungenslinien aufzuzeigen.

\section{Annäherungen an einen Forschungsüberblick zu Gender und Sprache in $\mathrm{DaZ}^{\star}$ (ab 2000)}

Gender als analytische Kategorie zu begreifen bedeutet, alle Facetten des Fachdiskurses Deutsch als Zweitsprache, der sich historisch aus dem Fach Deutsch als Fremdsprache entwickelt hat und der aktuell den sich etablierenden Diskurs um Sprachbildung (SB) in allen (schulischen) Fächern speist, auf Aspekte von Gender zu hinterfragen. Wo dies in theoretisch fundierter Weise im Bereich der Sprachdidaktik und Fremdsprachenforschung bisher geschehen ist, wird nachfolgend überblicksartig dargestellt.

\subsection{Gender in der Fremdsprachenforschung und Fremdsprachendidaktik}

Einen wichtigen Beitrag zum Einschreiben der zentralen Kategorie Gender in den Fachdiskurs der Fremdsprachendidaktiken leistet der Sammelband Gender Studies and Foreign Language Teaching (Decke-Cornill/Volkmann 2007a). Ausgehend von der Alltagsbeobachtung des Sprachenlernens als einer ,female domain" (Decke-Cornill/Volkmann 2007b: 7) und der Feststellung der Absenz des Themas im fremdsprachendidaktischen Diskurs stellt der Sammelband eine Bandbreite an Beiträgen zur Verfügung, deren Rezeption nötig ist, um zu einer aufgeklärten und fundierten Haltung gegenüber der Kategorie Gender zu gelangen. So wird Gender im Kontext von Globalisierung und Mehrsprachigkeit verortet (Piller/Pavlenko 2007), der Beruf der Sprachlehrerin (Haas 2007) und „das Sprachenlernen als Mädchensache“ (Doff/Klippel 2007) his- 
torisch betrachtet. Weitere Beiträge referieren überblicksartig verschiedene gendertheoretische Ansätze, Studien und Ergebnisse (vgl. Nelson 2007) oder widmen sich klassischen Fragen der Fremdsprachenforschung wie Interaktion und Gender (Decke-Cornill 2007), linguistischen Aspekten (Linke 2007), der Lehrkräftebildung (Dirks 2007) oder dem Potenzial der Literaturdidaktik (Volkmann 2007, Delanoy 2007 u. a.).

Der Beitrag von Barbara Schmenk (2007) soll an dieser Stelle ausführlicher dargestellt werden, da mit den Arbeiten von Schmenk (2002, 2007, 2009, 2016) besonders deutlich wird, wie die Reproduktion von unreflektiertem Alltagswissen und die Reproduktion sowie Perpetuierung stereotyper geschlechterdifferenzierender Praktiken in Lehre und Forschung zusammenhängen. Grundsätzlich, so Schmenk (2007), werden binäres Denken und Unterschiedlichkeiten der anatomischen Geschlechter (sex) stereotypisiert verwendet und so in empirische Untersuchungen zu Teilbereichen des Fremdsprachenlernens eingeschrieben. „Binary thinking and the quest for sex differences have traditionally dominated research into language learning/teaching. 'How do male and female learners differ?' has been the prevailing research paradigm in the field" (ebd.: 121). Am Beispiel der Faktoren Motivation und Lernstil zeichnet die Autorin nach, wie „weibliche“ und „männliche“ Lernende in binäre Kategorisierungen und Muster geordnet werden, und im Ergebnis Mädchen als die besseren Sprachlernenden erscheinen. Dies wiederum sieht Schmenk als ein Muster für die „feminization of language learning“ an, d. h. die Argumente empirischer Studien bestätigen Alltagshypothesen, verlassen jedoch nicht den Weg binär kategorisierender Zuordnungen, die wiederum auf tradierten Alltagshypothesen zum angeblichen Gegenpol „männlich/weiblich“ passen. Über den Versuch Unterschiede festzustellen entstehen Gendered images of motivation und Gendered images of learning styles (vgl. Schmenk 2007: 125ff.). Das Differenzparadigma ist nicht überwunden und Mädchen ,bleiben die besseren Sprachlernenden'.

Nach dem Erscheinen von Decke-Cornill/Volkmann (2007) gibt es mit Ausnahme der Monographie von Schmenk (2009) nur vereinzelt Publikationen zum Themenfeld Gender und Sprachen lernen, zumindest in Bezug auf deutschsprachige Publikationen. 2009 erscheinen das Themenheft Gender der Zeitschrift Praxis Fremdsprachenunterricht (ebenso unter der Leitung von Helene Decke-Cornill), einzelne Beiträge zur Lehrwerksanalyse (Maijala 2009) oder zu interkultureller Kommunikation und Geschlecht (Moghaddam 2009, Nowoczin 2009). Eichhof-Cyrus (2009) untersucht Sprachverwendung in DaF-Lehrwerken, Boysen/Michalak (2010) fragen, wie Jungen zum Lernen der Zweitsprache Deutsch motiviert werden können. Ortner (2010a, 2010b) beschreibt gendernormkritische Experimente sowie genderkritische 
Lehrwerksanalysen für den DaF-Unterricht. Starck (2011) stellt unterrichtspraktische Ansätze für den Englischunterricht vor, Gutenberg (2013) fordert grundsätzlich mehr Beschäftigung mit Gender und queer theories in den Fremdsprachendidaktiken, Kollig/Johnstone (2014) widmen sich audiovisuellen Medien und Gendervielfalt im DaF-Unterricht. Im Jahr 2015 erscheinen sowohl das bereits genannte Themenheft „Negotiating Gender“ (König/Suhrkamp/ Decke-Cornill 2015a) als auch ein umfassender Sammelband zur Auseinandersetzung mit Gender in schulischen Kontexten mit dem Titel „Teaching Gender?" (Wedl/Bartsch 2015). Letzterer verhandelt zahlreiche Aspekte schulischer Bildung genderreflexiv, theoretisch fundiert und praxisbezogen. Die drei enthaltenen Beiträge zu Gender und Fremdsprachenunterricht beziehen sich jedoch alle auf den Englisch bzw. Englisch- und Französischunterricht (vgl. König 2015, Lewin 2015, Mittag 2015).

Mit dem Themenheft „Negotiating Gender“ (König/Suhrkamp/DeckeCornill 2015a) wird der aufgeklärten und wissensbasierten Thematisierung von Gender im Englischunterricht ein hohes Potenzial für das Lernen der Fremdsprache zugesprochen. Der (schulische) Fremdsprachenunterricht wird als Ort und Raum gesetzt, in dem Genderreflexionen besonders gut bewerkstelligt werden können. Die Vermutung der Autorinnen, für die zusätzlich Belege aus Fallstudien zu Identitätsentwicklung und Fremdsprachenlernen herangezogen werden können (vgl. Sunderland 2000), lautet:

Hier kann der Zugang über die Fremdsprache ein Vorteil sein: Durch sie werden Aushandlungen über Geschlecht zwar häufig verlangsamt und in ihrer Komplexität reduziert, aber ihre Fremdheit kann auch eine Distanzierung von der Unmittelbarkeit der eigenen Sprachumgebung und damit ein freieres Sprechen als in der Erstsprache begünstigen. Gerade Themen und Begriffe, die emotional besetzt, tabuisiert oder anderweitig besonders normativ aufgeladen sind, lassen sich leichter in einer Sprache verhandeln, in der die sprachliche Ausdrucksform noch nicht so eng mit kultureller Bedeutung verknüpft ist. (König/ Suhrkamp/Decke-Cornill 2015b: 4)

Die bis hier gegebene Darstellung hatte eher aufzählenden Charakter und damit die Funktion, eine (historisch zurückweisende) Narrationslinie aufzuzeigen, an die angeknüpft werden kann. Es wurde gezeigt, dass vereinzelt, Gender und Sprache(n) lernen' in den Fremdsprachdidaktiken sowie in der Fremdund Zweitsprachenforschung thematisiert wird. Der nachfolgende Abschnitt nähert sich dem Themenfeld aus theoretischer Perspektiven und stellt dar, wo und wie Gender als analytische Kategorie in Bezug auf Sprache(n) lernen verortet sein kann ohne das Differenzparadigma fortzuschreiben. 


\subsection{Gender und Sprachgebrauch (lernen)}

Anknüpfend an die Hypothese von König/Suhrkamp/Decke-Cornill (2015b), dass das Lernen von Englisch als Fremdsprache besonders geeignet sei Genderreflexionen anzuregen, wird, ähnlich optimistisch, in einer Veröffentlichung des österreichischen Bundesministeriums für Unterricht, Kunst und Kultur (2011) die Rolle des beginnenden Spracherwerbs für beginnende Genderreflexionen im Rahmen schulischer Lernprozesse herausgehoben. Lehrkräfte sollten dies nutzen um geschlechtergerechtes Sprachverhalten zu vermitteln:

Die Initiierung geschlechtergerechten Sprachverhaltens müsste gerade beim Sprachenlernen eine zentrale Stellung einnehmen, da bei Neuerwerb einer Sprache die Chance gegeben ist, von Beginn an geschlechtergerechte Formulierungen zu verinnerlichen und nicht wie bei der Muttersprache derzeit üblich, sozialisierte Sprachformen erst hinterfragt und umgelernt werden müssen. Darüber hinaus kann das Erlernen geschlechtergerechter Formulierungen in der Fremdsprache dazu anregen, den umgangssprachlichen Gebrauch der Muttersprache kritisch zu reflektieren. (Bundesministeriums für Unterricht, Kunst und Kultur 2011: 71)

Auf konkrete Kontexte des Deutschlernens $\mathrm{DaZ} / \mathrm{DaF}$ übertragen, ergäbe sich mit jedem Anfangsunterricht die Möglichkeit, genderneutral, gendersensibel, genderkritisch oder genderreflexiv sprachlich zu handeln und dieses Handeln zu erlernen, unabhängig davon, inwiefern dies im Kontext der anderen Sprachen der Lernenden üblich ist. Entsprechende Vorschläge für geschlechtergerechtes Sprachverhalten oder auch gendersensiblen Sprachgebrauch liefern Gender Studies, feministische Linguistik und Gender Mainstreaming-Maßnahmen (vgl. z. B. AG Feministisch Sprachhandeln 2014/2015, Gäckle 2013, Stadt Wien 2011). Diese betrachten u. a. die Oberfläche der sprachlichen Mittel und Äußerungen und bieten Formulierungshilfen, alternative Formulierungsvorschläge und genderreflexiv passende Antworten auf potenziell diskriminierende Fragen an.

Das Verhältnis von Sprachgebrauch und Gender ist jedoch komplex und geht über die Verwendung genderreflexiver Formulierungsvarianten weit hinaus. Karin Wetschanow (2008) versucht, dieses umfassende Verhältnis zu bestimmen, obwohl es

(...) kein einfaches (ist), weil es uns immer über den Weg der Realität führt. (...) Dass „Geschlecht" uns überhaupt etwas und was es uns bedeutet, daran ist 
Sprache maßgeblich beteiligt. Sprache ist ein soziales und gesellschaftliches Phänomen und "Geschlecht" ist die Kategorie, die unser gesellschaftliches Zusammenleben ganz zentral strukturiert. Dass wir und wie wir über "Geschlecht“ sprechen, und dass wir und wie wir „Geschlecht“ leben und erleben hängt unmittelbar damit zusammen. (ebd.: 211)

Ausgangspunkt ihrer Darstellung sind die „unterschiedlichen Denkrichtungen und Überlegungen, die im Spannungsfeld ,Sprache' und ,Geschlecht ${ }^{`} \mathrm{zu}$ finden sind bzw. die eine linguistische Beschäftigung mit diesem Thema beeinflusst haben" (ebd.: 195). Dabei ist die theoretische Überlegung des Verhältnisses von Sprache und (sozialer) Wirklichkeit besonders wichtig. Zwei grundsätzliche Gegenpositionen beeinflussen unser Verständnis von Sprache und Gender nach wie vor: Erstens die Annahme, Sprache sei ein Abbild der Wirklichkeit, oder zweitens, Sprache sei eine die Wirklichkeit konstruierende Größe. Wird Sprache als Abbild von Realität und unveränderliches Sprachsystem verstanden, ist im Sinne der Abbildtheorie, Geschlecht ${ }^{\varsigma}$ unabhängig von Sprache vorhanden, wird über Wahrnehmung und Erfahrung vermittelt und ist in Sprache vorhanden. „Konkret würde das bedeuten: Es gibt zwei Geschlechter, die wir täglich wahrnehmen und in der Sprache mit Begriffen wie ,weiblich` und ,männlich', oder ,Frau' und ,Mann“ abbilden“ (ebd.: 196).

Im Gegensatz dazu steht die konstruktivistische Annahme von Sprache als einer Größe, die die Wirklichkeit konstruiert. Unsere „Wahrnehmung der Welt (ist) nachhaltig von Sprache geprägt (...)“, bzw. Sprache konstruiert unsere wahrgenommene Wirklichkeit (ebd.: 197). Denken ist ohne Sprache nicht möglich und von der Wahrnehmung sprachlicher Handlungen abhängig. Die Austinsche Sprechakttheorie weiter entwickelnd hat Judith Butler in Gender Trouble (1990) „eine konstruktivistische, diskurstheoretische Sichtweise des Geschlechterverhältnisses" etabliert, in der die Binarität der Geschlechter als essentialisierte Konstruktion und Geschlechterdifferenz als diskursiv naturalisiert gelten. Geschlechterdifferenz existiert demnach nicht einfach, sie ist eine „sprachlich hergestellte Kategorisierung, die uns lediglich als selbstevident und unhinterfragbar, d. h. natürlich erscheint. Die Geschlechterdifferenz selbst wird als soziale Konstruktion angenommen, als Effekt des Diskurses“ (ebd.: 197). Sprache wird demnach als „,wichtiges und machtvolles Mittel der Herstellung von ,Geschlecht ${ }^{6}$ angenommen und es wird davon ausgegangen, dass durch die Auswahl sprachlicher Mittel (linguistic choice), Gender' aktiv hergestellt wird“ (ebd.: 198). ${ }^{1}$

1 Vertiefend zum Verhältnis von Sprache und Gender aus strukturalistischer und konstruktivistischer Sprachsicht vgl. z. B. Hornscheidt (2013). 
Bezogen auf das Lernen und Erwerben einer neuen Sprache könnte hier durch die gendersensible Wahl der sprachlichen Mittel auf der sprachlichen Oberfläche eine veränderte Wahrnehmung von Geschlecht/Gender bei Lernenden angelegt werden, die sich dann eine neue gendersensible oder geschlechtergerechte Realität diskursiv konstruieren können. D. h., durch die Verwendung geeigneter, genderreflexiver Konstruktionshilfen (wie sie z. B. in den o. g. Handreichungen angeboten werden) können wir auch zur Reflexion über Gender-Verhältnisse und die Bedeutung der Sprache darin anregen. Belege für den positiven Effekt der Verwendung geschlechtergerechterer Sprache liegen zum Beispiel aus der Bildungspsychologie vor (vgl. Vervecken/Hannover 2015)2. Die paarformige Bezeichnung von Berufen beispielsweise ist in Deutschlehrwerken in der Regel bereits Standard. Zukünftig ist es jedoch von Bedeutung von der Wortebene auf die Ebene von Texten, Argumentationen und Perspektivierungen zu schauen, in denen Klischeehaftes, Stereotypisierendes, Belustigendes oder gar Herabwürdigendes im Sprachgebrauch durch ihre Gegenteile ersetzt werden bzw. Aspekte von Gender in ihrer sprachlichen Verwobenheit mit anderen Kategorien auch im Unterricht reflektiert werden (vgl. dazu Peuschel/Dirim in Vorb.). ${ }^{3}$

Lann Hornscheidt (2012) wirft aus feministisch linguistischer Perspektive im Weiterdenken zur Macht von Sprache und Sprachgebrauch kontroverse und eher selten gestellte Fragen auf, die an dieser Stelle nicht ausgelassen

2 Verhecken/Hannover (2015) testen den Einfluss einer sprachlichen Intervention - die Verwendung paarformiger Berufsbezeichnungen bei als typisch männlich konnotierten Berufen (z. B. Feuerwehrmann/Feuerwehrfrau, Ingenieurin/Ingenieur, Geburtshelferin/ Geburtshelfer) - auf die Selbstwirksamkeit von 591 niederländischen und deutschen Grundschulkindern. Ein Ergebnis der Studie ist, dass die Vorstellung, einen bestimmten Beruf ergreifen zu können, durch die Art und Weise der gegenderten Bezeichnung beeinflusst wird. Demnach trauen sich Mädchen viel eher zu, einen bestimmten männlich konnotierten Beruf zu erlernen, wenn dieser auch in der weiblichen Berufsbezeichnung angeboten wird: „Two classroom experiments (...) showed that the presentation of occupational titles in pair forms (...), rather than in generic masculine forms (...), boosted children's self-efficacy with regard to traditionally male occupations, with the effect fully beeing mediated by perceptions that the jobs are not as difficult as gender stereotypes suggest" (ebd.: 76).

3 Decke-Cornill (2015: 10) schlägt hierfür methodisch verschiedene Vorgehensweisen vor, so z. B. das Ent/Dramatisieren der zweigeschlechtlichen Ordnung „durch Aufgaben, die das vermeintlich ,Typische' von Geschlechteridentitäten normalisieren“ (ebd.), das Schaffen von Aushandlungs- und Verständigungsgelegenheiten, das Überarbeiten von Texten und Formulierungen mit gendergerechten sprachlichen Mitteln und die Präsentation nonkonformer Figuren in Texten und Filmen. 
werden sollen. Die Beschäftigung mit der Rolle von Sprachgebrauch für die Dekonstruktion binärer Geschlechterverhältnisse wird rückgebunden an die wandelbare Verfasstheit des Sprachsystems selbst. Es werden Beziehungen gezogen zu sprachlich vermittelten Ein- und Ausschlussmechanismen auf Grund von Sprachkompetenz und Deutsch als Zweitsprache. Hornscheidt verbindet Aspekte von Diskriminierung mit Sprachgebrauch, die wiederum mit der Frage nach dem Umgang mit (sprachlicher) Heterogenität, Bildungsbenachteiligung und dem Lernen des Deutschen als Zweit- und Fremdsprache zusammenhängen. Ausgehend von der Annahme der radikalen Konstruktion von Wirklichkeit durch Sprache schreibt Hornscheidt:

es gibt keine sprache hinter dem sprechen, sondern nur besonders stark konventionalisierte formen von sprachhandlungen, die sozial normiert sind und auf gesellschaftlich machtvollen übereinkünften beruhen - und diese gesellschaftlichen normierungen auch immer wieder re_produzieren und damit auch für ausschlüsse und machthierarchien sorgen. warum beispielsweise gibt es noch genusformen im deutschen? warum gibt es so viele verkomplizierende rechtschreibereformen (sic!) aber keine nachhaltigen bestrebungen, deutsche normierte schreib- und sprechsprache so zu verändern, dass personen, die deutsch nicht als erstsprache verwenden, leichter zugang dazu haben? warum geht eine normierte sprachnorm des deutschen davon aus, dass unterschiedlich migratisierte varianten nicht der norm entsprechen - sowohl im sprechen als auch im schreiben - warum wird die normfeststellung nicht verändert stattdessen und gesellschaftlichen verhältnissen und den spre_cherinnen angepasst, sondern müssen sich die sprech_erinnen einer fiktiven und machtvollen sprachnorm anpassen - dadurch würde eine ganze andere grundlage einer sprachlichen identifizierung für verschiedene personen und personengruppen ermöglicht, denen sprache dann gehören würde als handlungsform und die nicht gemessen würden an einer privilegierten abgehobenen sprachnorm. geht es in der aufrechterhaltung von normsprachen also vielleicht um mehr und um anderes als ausschließlich um die ermöglichung von kommunika-tion? geht es auch um grenzziehungen, um ausschlüsse, um kontrolle, um bestandssicherung, um ausbeutung und diskriminierung?(sic!) (ebd.: 40)

In den Kategorien Sprache, Geschlecht und natio-ethno-kulturelle Zugehörigkeit (vgl. Mecheril 2016: 15), die in sich jeweils äußert heterogen sind, lassen sich vergleichbare Machtprinzipien und Differenzsetzungen feststellen. Situativ und individuell hergestellt können die drei genannten Differenzlinien miteinander in Beziehung gesetzt werden. Im nächsten Abschnitt werden daher Sprache/Zweitsprache und Gender als zwei von zahlreichen Differenz- 
linien individuell-gesellschaftlicher Positionierungsmöglichkeiten dargestellt sowie Intersektionalität als mögliche Perspektive für theoretische und empirische Zugänge zu Gender und Deutsch als Zweit- und Fremdsprache zur Diskussion gestellt.

\subsection{Differenzlinien (Zweit)Sprache und Gender - Intersektionalität als Perspektive}

Geschlecht und Sprache werden als zwei von zahlreichen möglichen bipolaren Differenzlinien angesehen, über die Individuen sozial markiert und positioniert sind (vgl. Leiprecht/Lutz 2015: 287). Mit dem Begriff Differenzlinie gehen die Autorinnen davon aus, „dass bestimmte soziale Unterschiede und Unterscheidungen in der Gesellschaft wirkungsmächtig vorhanden sind (...)“ (ebd.: 285). Sowohl Sprache als auch Geschlecht werden als bipolare Kategorien angenommen, die unter der Leitkategorie Diversität (oder auch Heterogenität) verhandelt werden. In der Darstellung der 15 bipolaren Differenzlinien nach Lutz finden sich außer Sprache und Geschlecht, das den ersten Platz in der Tabelle einnimmt, noch die Kategorien (bzw. Differenzlinien) Sexualität, ,Rasse'/Hautfarbe (mit einer erklärenden Fußnote zur Verwendung der umstrittenen Begrifflichkeiten), Ethnizität, Nation/Staat, Klasse/ Sozialstatus, Religion, Kultur, Körper, Generation, Sesshaftigkeit/Herkunft, Besitz, Nord-Süd/Ost-West sowie Gesellschaftlicher Entwicklungsstand (vgl. Leiprecht/Lutz 2015: 287). Dabei wird ein Grunddualismus aller Kategorien angenommen, der sich in eine dominierende Seite und eine dominierte Seite teilt. Die Herrschaftsverhältnisse in der Differenzlinie ,Sprache sind mit „überlegen“ vs. und „unterlegen“ markiert, diejenigen in der Linie ,Geschlecht' mit „männlich/zweigeschlechtlich“ vs. „weiblich/transgender“ (ebd.). Zur Binarität der herkömmlichen Gendervorstellungen wird hier die Abgrenzung des Systems der Zweigeschlechtlichkeit von anderen Konzepten (und Realitäten), z. B. transgender, vorgenommen.

Der Gedanke (diskriminierender) Intersektionen hat seinen Ursprung in der US-amerikanischen feministischen Diskussion der 1990er Jahre. Er soll hier für Gender und Sprache in DaZ* als theoretischer Ausgangspunkt aufgegriffen werden, weil nicht die Kategorien an sich bedeutsam für Diskriminierungen sind bzw. waren, sondern ihre hinderlichen Überkreuzungen und individuellen Überschneidungen (vgl. Walgenbach 2012).

Ausgangspunkt dieser Argumentation ist die Feststellung, dass alle Menschen sozusagen am Schnittpunkt (intersection) dieser Kategorien positioniert sind und dort ihre Loyalitäten und Präferenzen entwickeln. Die Kategorien sind also 
nicht nur soziale Platzanweiser, sondern sie generieren auch Identität. Die Herausforderung an Theorie und Empirie ist nun, nicht nur von der Komplexität der Differenzlinie zu sprechen, sondern Instrumente zu entwickeln, mit denen diese Komplexität als Verhältnis untersucht und fassbar gemacht werden kann (...). (Leiprecht/Lutz 2015: 288)

Melter/Schäfferlin (2016: 284) stellen unter der Perspektive von Intersektionalität die „Konstruktion von Geschlechtern (...) oftmals mit migrationsgesellschaftlichen und rassistischen Vorstellungen verwoben“ dar, die nicht additiv, sondern in ihren Verflechtungen und Wechselwirkungen zu untersuchen ist (ebd.). Die bei Melter/Schäfferlin aufgeführten Studien, die die bislang ,eher getrennte Thematisierung von ,Gender und Bildung' auf der einen und ,Migration und Bildung' auf der anderen Seite" (ebd.: 286) aufheben, stammen jedoch nicht aus dem Kontext des Sprachenlernens. Hier besteht Handlungs- und Forschungsbedarf für unsere Fächer, wobei durchaus an Untersuchungen vergangener Jahre zum Themenfeld Mehrsprachigkeit, Zweitspracherwerb und Gender angeknüpft werden kann (vgl. Pavlenko et al. 2001). Die aktuellen Ansätze machtkritischer Perspektivierungen der „Sprachverhältnisse“ in migrationsgesellschaftlichen Zusammenhängen liefert Dirim (2016). Diese können eine Grundlage für Untersuchungen zur Verwobenheit von Genderaspekten und dem Sprachenlernen (des Deutschen) bilden.

\subsection{Zusammenfassung}

Der vorhergehende Abschnitt 3 hatte das Ziel, die Kategorien Gender und Sprache in alten und neuen Zusammenhängen in Bezug auf den interdisziplinären und heterogenen Fachdiskurs im Fach DaZ* exemplarisch darzustellen. Dafür wurden fremd- und zweitsprachendidaktische Studien, bildungswissenschaftliche Befunde, linguistische Ansätze und Ansätze der Gender Studies herangezogen. Es ist deutlich geworden, dass Gender und Sprache in vielen Bezugsdisziplinen und Bezugsdiskursen für das Fach DaZ* eine Rolle spielen und als Kategorien existieren. Es ist auch deutlich geworden, dass je nach Disziplin die Kategorien unterschiedlich gefasst und ausgestaltet werden. Während in der Fremdsprachendidaktik Gender als Thema und in Bezug auf die Reflexion sprachlichen Handelns betont wird, spielen Gender und (Zweit)Sprache differenzparadigmatisch gedacht nur als Indikatoren für andere, hierarchisch übergeordnete Kategorien wie Bildungserfolg eine Rolle. Postmoderne feministische Ansätze stellen Gender und Sprache nicht getrennt voneinander dar, sondern als füreinander notwendiges, sich bedingendes Repertoire für das Sprechen über Machtverhältnisse. Dabei wird die 
Veränderlichkeit der Machtverhältnisse durch die Veränderlichkeit der Sprache positiv bewertet. Im Ansatz der Intersektionalität werden analytische Kategorien benannt und es wird dazu aufgefordert, ihre Verwobenheit zu untersuchen und Erkenntnisse für professionelles nichtdiskriminierendes Lehrhandeln zu gewinnen. Ganz gleich, aus welcher Perspektive wir uns dem Themenfeld nähern - Gender ist präsent und hat mit Sprache und damit auch mit dem Sprache(n) lernen zu tun.

Im nachfolgenden und letzten Abschnitt dieses Beitrages soll nun explizit gemacht werden, welche Verbindungen zwischen Gender und Sprache im Feld DaZ* zukünftig zu berücksichtigen sind.

\section{Gender und Sprache im Feld DaZ* - Desiderata}

Leiprecht/Lutz (2015: 285) fordern: „Bei der Thematisierung von sozialer Heterogenität muss die bisher noch weitverbreitete Praxis einer jeweils exklusiven Bearbeitung von Differenzlinien in ,Spezialpädagogiken', wo entweder zu sozialer Klasse oder zu Geschlecht oder zu Ethnizität gearbeitet wird, überwunden werden“. Gleichzeitig gilt es, die vorhandene Expertise der sog. Spezialpädagogiken aufzugreifen, und sie weder zu vergessen noch zu verdrängen. Vielmehr ist es wünschenswert, interdisziplinär und intersektional zu denken und zu forschen. So können die hier als relevant gesetzten Differenzlinien, die bisher entweder dethematisiert werden (Gender) oder zu einseitig betrachtet werden (Zweitsprache als Defizit), miteinander in Beziehung gesetzt werden und ihre individuelle und gruppenspezifische Verwobenheit untersucht werden. Es besteht dank der zunehmenden Beschäftigung mit der Rolle von Sprache für fachliches Lernen in schulischen Kontexten die Möglichkeit der Normalisierung sprachbewussten und sprachsensiblen Handelns. Zudem scheint es lohnenswert, systematisch Fragen zu stellen, deren Beantwortung intersektionales Denken erfordert und die das Lehren und Lernen von Deutsch als Zweit- und Fremdsprache an den Schnittpunkten von Sprache und Gender erfassen. Aber auch komplexe Untersuchungen zu weiteren Differenzlinien wie Alter, ,Behinderung', sozialer Status etc. sind wünschenswert. Zu einigen hier genannten Differenzlinien können bereits umfangreiche Forschungsergebnisse der Zweit- und Fremdsprachenforschung herangezogen werden, so z. B. zum (Faktor) Alter im Zweitsprachenerwerb, die jedoch (macht)kritisch hinterfragt werden müssten. Zukünftige intersektionale Forschungsarbeiten zu Gender und Sprache in DaZ* stehen vor der Herausforderung, Mindeststandards der Theoriebildung für die drei Differenzlinien 
race, class, gender zu erfüllen (vgl. Leiprecht/Lutz 2015: 290f.), die alle als situativ ausformulierte gesellschaftliche Makrofaktoren gelten, welche gleichzeitig sozial positionieren wie auch als Platzanweiser wirken. Sie werden durch das „doing“ kontinuierlich reproduziert und normalisiert, gleichzeitig haben sie die Funktion der Identifikation durch Selbstwahrnehmung bzw. Fremdzuschreibung. Ein Grundverständnis für Sprache, das den vorgenannten Kategorien hinzugefügt werden kann, beinhaltet, dass Sprachaneignungsprozesse in individuellen Lebensverläufen zwar unterschiedlich verlaufen, jedoch stets stattfinden, eingebettet in soziale, von Machtverhältnissen strukturierte Zusammenhänge.

Neben den Herausforderungen an die Theoriebildung potenziell machtkritischer und intersektional arbeitender Forschungstätigkeit zu Gender und Sprache in DaZ* erscheinen vor allem Untersuchungen von Unterrichtsdiskursen, beispielsweise des sprachförderlichen schulischen Unterrichts hinsichtlich intersektional wirkender Zuschreibungen sowie die Analyse von zuschreibungsintensiven, potenziell diskriminierenden Unterrichtsmaterialien (vgl. Dirim 2015, Peuschel/Dirim in Vorb.) interessant für die weitere Auseinandersetzung.

Die bis hier vorgenommenen wechselseitigen Bezugnahmen und Annäherungen von Sprache und Gender für das Lernen und Lehren des Deutschen als nicht-erster Sprache machen Folgendes deutlich: Gender und (Zweit)Sprache sind soziale Konstruktionen und als solche sind sie veränderbar. Während bei der Beschäftigung mit Zweit- und Fremdsprachenerwerb die Annahme der Veränderlichkeit sprachlicher Kompetenzen eine Voraussetzung für die Existenz unserer Fächer ist, sind wir von der Normalisierung nicht-binärer, dynamischer und nicht heteronormativer Gender-Konstruktionen weit entfernt. Während wir wissen, dass Menschen nicht mit einer Sprache geboren werden, sondern die sprachlichen Repertoires im Laufe ihres Lebens erlangen und lebensweltliche Mehrsprachigkeit zumindest als Begriff etabliert ist, sind wir von der Akzeptanz lebensweltlicher Mehrgeschlechtlichkeit in Alltagswissen und Fachdiskurs weit entfernt.

Ziel des Beitrages war es, innerhalb neu verhandelter normativer Grundlagen und reflektierter Praktiken Ideen zur Verknüpfung von Gender und Sprache für das Lernen und Lehren von Deutsch als nicht-erster Sprache zu erarbeiten. Überblicksdarstellungen sind dabei der Beginn vertiefter Auseinandersetzung mit postkolonial argumentierenden, migrationspädagogischen und genderkritischen Positionen im Fachdiskurs. Der Beitrag möge Anregungen liefern, sich der Kategorie Gender gleichberechtigt neben anderen Kategorien anzunähern und Gender zukünftig stärker mit diesen verwoben zu denken, zu analysieren und zu diskutieren. 


\section{Literatur}

AG Feministisch Sprachhandeln der Humboldt-Universität zu Berlin (2014/2015, 2. Auflage): Was tun? Sprachhandeln - aber wie? W_ortungen statt Tatenlosigkeit! Berlin: Humboldt-Universität (online: http://feministisch-sprachhandeln.org, letzter Aufruf: 11.1.2017).

Bausch, Karl-Richard; Christ, Herbert; Krumm, Hans Jürgen (2007) (Hrsg.): Handbuch Fremdsprachenunterricht. Tübingen, Basel: Francke.

Bartsch, Annette/, Julia (2015): Teaching Gender? Zum reflektierten Umgang mit Geschlecht im Schulunterricht und in der Lehramtsausbildung. In:/Bartsch (Hrsg.).

S. 9-31.

Boysen, Julia/Michalak, Magdalena (2010): Geschlechtsspezifische Differenzierung im Förderunterricht: Wie motiviert man Jungen, die Zweitsprache zu lernen?. In: Deutsch als Zweitsprache 2010, 1, S. 23-32.

Braun, Cordula/Schwemer, Kai (2013) (Hrsg.): Themenheft „Jungen fördern“. Der fremdsprachliche Unterricht. Französisch 47/122 (2013).

Bundesministerium für Arbeit, Soziales und Konsumentenschutz (2010): Leitfaden für diskriminierungsfreie Sprache, Handlungen, Bilddarstellungen. Wien. (online: https://www.schule.at/portale/gender-und-bildung/materialien/gendersensible-nichtdiskriminierende-sprache/detail/leitfaden-fuer-diskriminierungsfreie-sprache-handlungen-und-bilddarstellungen.html, letzter Aufruf: 11.1.2017).

Burwitz-Melzer, Eva/Mehlhorn, Grit/Riemer, Claudia/Bausch, Karl-Richard/Krumm, Hans-Jürgen (Hrsg.): Handbuch Fremdsprachenunterricht (6., völlig überarbeitete und erweiterte Auflage). Tübingen: A. Francke Verlag.

Butler, Judith (2009): Gender Trouble. Feminism and the Subversion of Identity. New York, London: Routledge.

Decke-Cornill, Helene (2007): The Issue of Gender and Interaction in the L2-Classroom. In: Decke-Cornill/Volkmann (Hrsg.), S. 77-90.

Decke-Cornill, Helene (2009) (Hrsg.): Themenheft "Gender". Praxis Fremdsprachenunterricht. Die Zeitschrift für das Lehren und Lernen fremder Sprachen 6/2009. Oldenburg: Cornelsen.

Decke-Cornill, Helene/Volkmann, Laurenz (2007a) (Hrsg.): Gender Studies and Foreign Language Teaching. Tübingen: Narr.

Decke-Cornill, Helene/Volkmann, Laurenz (2007b): Introduction. In: Decke-Cornill/ Volkmann (Hrsg.), S. 7-14.

Decke-Cornil (2015): Differenzsensibel unterrichten. In: König/Suhrkamp/DeckeCornill (Hrsg.), S. 10.

Delanoy, Wener (2007): Gender and Literature Didactics. In: Decke-Cornill/Volkmann (Hrsg.), S. 185-208. 
Dirim, İnci (2015): Hochschuldidaktische Interventionen: DaZ-Lehrende und Studierende eruieren Spielräume machtkritisch positionierten Handelns. In: Thoma, Nadja/Knappik, Magdalena (2015) (Hrsg.): Sprache und Bildung in Migrationsgesellschaften: machtkritische Perspektiven auf ein prekarisiertes Verhältnis. Bielefeld: transcript, S. 299-316.

Dirim, İnci (2016): Sprachverhältnisse. In: Mecheril, Paul (Hrsg.) unter der Mitarbeit von Veronika Kourabas und Matthias Raugger. Handbuch Migrationspädagogik. Weinheim, Basel: Beltz. S. 311-325.

Dirks, Una (2007): The Professional Development of CLIL Teachers in Relation to Their Gender-Specific Ressources. In: Decke-Cornill/Volkmann (Hrsg.), S. 103120.

Doff, Sabine/Klippel, Renate (2007): Mädchen lernen fremde Sprache - Lehrbücher und Lektüre im 18. und 19. Jahrhundert. In: Decke-Cornill/Volkmann (Hrsg.), S. 47-62.

Eichhoff-Cyrus, Karin M. (2009): Feministische Linguistik im Unterricht 'Deutsch als Fremdsprache'. In: Lochtman, Katja/Müller, Heidy Margrit (Hrsg.): Sprachlehrforschung: Festschrift für Prof. Dr. Madeline Luthejharms. Bochum: AKS-Verlag. S. $125-135$.

Elsner, Daniela/Lohe, Viviane (2016) (Hrsg.): Gender und Language Learning - Research and Practice. Tübingen: NarrFranckeAttempto.

Faulstich-Wieland, Hannelore/Weber, Martina/Willems, Katharina (20092): Doing Gender im heutigen Schulalltag. Empirische Studien zur sozialen Konstruktion von Geschlecht in schulischen Interaktionen. Weinheim. Beltz Juventa.

Fleßner, Heike (2015): Geschlechterbewusste Pädagogik im Kontext diversitätsbewusster Pädagogik. In: Leiprecht, Rudolf/Steinbach, Anna (Hrsg.): (Band 1), S. 305-323.

Gäckle, Annelene (2013): ÜberzeuGENDERe Sprache - Leitfaden für eine geschlechtersensible und inklusive Sprache (Gleichstellungsbeauftragte der Universität zu Köln). Köln. (online: https://www.dshs-koeln.de/fileadmin/redaktion/Hochschule/Gender_und_Diversity/Sprachleitfaden.pdf, letzter Aufruf: 11.1.2017).

Gutenberg, Andrea (2013): Gendering/Queering the Language Classroom - Gender und Queer Studies als Herausforderung für das schulische Fremdsprachenlernen. In: Kleinau, Elke/Schulz, Dirk/Völker, Susanne (Hrsg.) Gender in Bewegung Aktuelle Spannungsfelder der Gender und Queer Studies. Bielfeld: transcript.

Haas, Renate (2007): Language Teaching as a „Women's job“: Historical and Current Perspectives. In: Decke-Cornill/Volkmann (Hrsg.), S. 31-46.

Hornscheidt, Lann (2012): feministische w_orte: ein lern-, denk- und handlungsbuch zu sprache und diskriminierung, gender studies und feministischer linguistik. Frankfurt a.M.: brandes \& apel. 
Hornscheidt, Lann (2013) Sprache/Semiotik. In: von Braun, Christina/Stephan, Inge (Hrsg.): Gender@Wissen - Ein Handbuch der Gender-Theorien. Köln, Weimar, Wien: Böhlau Verlag, UTB. S. 343-364.

Kollig, Danielle Verena/Johnstone, Japhet (2014): ,Queer Media': (Audio)-Visuelle Medien und Gender-Vielfalt im DaF-Unterricht. In: Hieronimus, Marc (Hrsg.). Visuelle Medien im DaF-Unterricht (Materialien Deutsch al Fremdsprache, 90). Göttingen: Universitätsverlag, S. 401-420.

König, Lotta (2015): Teaching Gender Reflection! - Theoretische Grundlagen und literaturdidaktische Unterrichtsbeispiele für einen genderreflektierenden Englischunterricht. In: Wedl/Bartsch (Hrsg.), S. 161-288.

König, Lotta/Suhrkamp, Carola/Decke-Cornill, Helene (2015a): Themenheft „Negotiating Gender", Der Fremdsprachliche Unterricht. Englisch 135/2015.

König, Lotta/Suhrkamp, Carola/Decke-Cornill, Helene (2015b): Negotiating Gender - Aushandlungs- und Reflexionsprozesse über Geschlechtervorstellungen im Fremdsprachenunterricht anstoßen. In: Der Fremdsprachliche Unterricht. Englisch 135/2015, S. 2-8.

Krumm, Hans-Jürgen/Fandrych, Christian/Hufeisen, Britta/Riemer, Claudia (2010) (Hrsg.): Deutsch als Fremd- und Zweitsprache - Ein internationales Handbuch. Berlin u. a.: De Gruyter.

Leiprecht, Rudolf/Lutz, Helma (2015): Intersektionalität im Klassenzimmer: Zur sozialen Konstruktion und Bedeutung von Ethnie, Klasse, Geschlecht und ihren Verbindungen. In: Leiprecht/Steinbach (Hrsg.), (Bandd. 1)., S. 283-302.

Leiprecht, Rudolf/Steinbach, Anna (2015) (Hrsg.) Schule in der Migrationsgesellschaft - Ein Handbuch (2 Bände). Schwalbach: Debus Pädagogik Verlag.

Lewin, Sonja (2015): Mit Bildern das Thema Gender bearbeiten - Praxisanregungen für den Englisch- und Französischunterricht der Sekundarstufe II. In: Wedl/ Bartsch (Hrsg.), S. 289-310.

Linke, Gabriele (2007): Linguistic Aspects of Gender in the Foreign Language Classroom. In: Decke-Cornill/Volkmann (Hrsg.), S. 137-160.

Mecheril, Paul (2004): Einführung in die Migrationspädagogik. Weinheim, Basel: Beltz.

Mecheril, Paul (2016) (Hrsg.) Mitarbeit von Veronika Kourabas und Matthias Rangger: Handbuch Migrationspädagogik. Weinheim, Basel: Beltz.

Melter, Claus/Schäfferlin, Stefan (2016): Geschlechterverhältnisse. In: Mecheril, Paul (Hrsg.) unter der Mitarbeit von Veronika Kourabas und Matthias Raugger. Handbuch Migrationspädagogik. Weinheim, Basel: Beltz. S. 277-293.

Maijala, Minna (2009): Typisch Frau ... Typisch Mann ...: Zur Genderproblematik in Lehrwerken für Deutsch als Fremdsprache. In: Zielsprache Deutsch 36, 1, S. 3360. 
Mittag, Martina (2015): Geschlecht als leerer Signifikant - Gendersensible Didaktik im Fremdsprachenunterricht. In: Wedl/Bartsch (Hg.), S. 251-260.

Moghaddam, Roy (2009): Interkulturelle Kommunikation und Geschlecht? In: Hunstiger, Agnieszka/Koreik, Uwe (Hrsg.) Chance Deutsch. Schule, Studium, Arbeitswelt - 34. Jahrestagung des Fachverbandes Deutsch als Fremdsprache 2006 (Materialien Deutsch als Fremdsprache, 78), Göttingen: Universitätsverlag, S. 513-522.

Nelson, Cynthia D. (2007): Queer Thinking about Language Teaching - An Overview of Published Work. In: Decke-Cornill/Volkmann (Hrsg.), S. 63-76.

Nowoczin, Jessica (2009): Dramenarbeit im Englischunterricht der Sekundarstufe I im Hinblick auf Gendersensibilisierung und interkulturelle Kommunikation. Kiel. Europäische Hochschulschriften, (Reihe 11 Pädagogik). Frankfurt a. M.: Lang.

Ortner, Rosemarie (2010a): Gender im Sprachunterricht? Normenkritische Experimente zwischen Sprachkurs und Gender-Training. In: Krumm, Hans-Jürgen/ Portmann-Tselikas, Paul R. (Hrsg.) Schwerpunkt: Lesen. Prozesse, Kompetenzen, Förderung (Theorie und Praxis. Österreichische Beiträge zu Deutsch als Fremdsprache, Seria A; 13/2009. Insbruck: Studienverlag, S. 195-208.

Ortner, Rosemarie (2010b): Mit der Vielfalt jenseits von Gendernormen rechnen: DaF-Lernende als AkteurInnen von Sprach- und ,Gender-Wandel' - Gendertheorie und Didaktik für den DaF-Unterricht. In: Schenk, Klaus/Masát, András (Hrsg.) Jahrbuch der ungarischen Germanistik 2009. Budapest, Bonn: DAAD, S. 240262.

Pavlenko, Aneta/Blackledge, Adrian/Piller, Ingrid/Teutsch-Dwyer, Marya. (Hrsg.) (2001): Mulitlingualism, Second Language Learning, and Gender. Berlin, New York: Mouton de Gruyter.

Peuschel, Kristina/Dirim, İnci (in Vorb.): Migrationspädagogische Vorschläge zum Umgang mit Sexismus in Lehrwerken für die Vermittlung des Deutschen als Zweitsprache. In: Heinemann, Alisha B./Khakpour, Natascha (Hrsg.): Sprachliche Gewalt und Geschlechterordnungen in der Migrationsgesellschaft. Heidelberg: Springer.

Piller, Ingrid/Pavlenko, Aneta (2007): Globalization, Gender and Multilingualism, In: Decke-Cornill/Volkmann (Hrsg.), S. 15-30.

Prenzel, Manfred/Sälzer/Christine/Klieme, Eckhard/Köller, Olaf (Hrsg.) (2013): PISA 2012. Fortschritte und Herausforderungen in Deutschland. Waxmann. (online: http://www.pisa.tum.de/fileadmin/w00bgi/www/Berichtband_und_Zusammenfassung_2012/PISA_EBook_ISBN3001.pdf, letzter Aufruf: 11.1.2017).

Schmenk, Barbara (2002): Fremdsprachenlernen - Frauensache? Einige Überlegungen zur Kategorie Geschlecht in der Fremdsprachenforschung. In: Zeitschrift für Fremdsprachenforschung 13, 2, S. 1-62. 
Schmenk, Barbara (2007): Foreign Language Research and the Feminization of Language Learning In: Decke-Cornill/Volkmann (Hrsg.), S. 121-136.

Schmenk, Barbara (2009): Geschlechtsspezifisches Fremdsprachenlernen? Zur Konstruktion geschlechtstypischer Lerner- und Lernbilder in der Fremdsprachenforschung. Tübingen: Stauffenburg.

Schmenk, Barbara (2016) Geschlecht. In: Burwitz-Melzer et al. (Hrsg.), S. 254-257.

Schneider, Claudia/Tanzberger, Renate/Traunsteiner, Bärbel. Bundesministerium für Bildung; Abt. Gender Mainstreaming/Gleichstellung und Schule (2011) (Hrsg.) (2011): Unterrichtsprinzip „Erziehung zur Gleichstellung von Frauen und Männern“ - Informationen und Anregungen zur Umsetzung ab der 5. Schulstufe. Wien. (online: http://pubshop.bmbf.gv.at/detail.aspx?id=192, letzter Aufruf: 11.1.2017).

Schößler, Franziska (2008): Einführung in die Gender Studies. Berlin: Akademie Verlag.

Sunderland, Jane (2000): Issues of language and gender in second and foreign language education. In: Language Teaching 33, S. 203-223 (online: doi 10.1017/ S0261444800015688)

Stadt Wien (2011): Leitfaden für geschlechtergerechtes Formulieren und eine diskriminierungsfreie Bildsprache (online: https:/www.wien.gv.at/medien/service/ medienarbeit/richtlinien/leitfaden-gender/, letzter Aufruf: 11.1.2017).

Starck, Kathleen (2011): „Yet the Details are not simple“- Teaching Gender? In: Linke, Gabriele (Hrsg.), Teaching Cultural Studies: Methods - Matters - Models. Heidelberg: Winter.

Vervecken, Dries/Hannover, Bettina. (2015). Yes I can! Effects of gender fair job descriptions on children's perceptions of job status, job difficulty, and vocational self-efficacy. In: Social Psychology 46, S. 76-92.

Volkmann, Laurenz (2007): Gender Studies and Literature Didactics: Research and Teaching - Worlds Apart?. In: Decke-Cornill/Volkmann (Hrsg.), S. 161-184.

Walgenbach, Katharina (2012): Intersektionalität - eine Einführung. Verlagsort: Verlag (online unter: http://portal-intersektionalitaet.de/theoriebildung/schluesseltexte/walgenbach-einfuehrung/, letzter Aufruf: 11.1.2017).

Wedl, Juliette/Bartsch, Annette (Hrsg.) (2015): Teaching Gender? Zum reflektierten Umgang mit Geschlecht im Schulunterricht und in der Lehramtsausbildung. Bielefeld: transcript.

Wetschanow, Karin (2008): Über das Verhältnis von Sprache und Geschlecht. In: Buchmayr, Maria (2008) (Hrsg.): Geschlecht lernen: gendersensible Didaktik und Pädagogik. Innsbruck (u. a.): StudienVerlag, S. 195-213. 